\title{
Persuasive Technology to Improve Eating Behavior using a Sensor-Embedded Fork
}

\author{
Azusa Kadomura $^{1} \quad$ Cheng-Yuan Li $^{2} \quad$ Koji Tsukada $^{3} \quad$ Hao-Hua Chu $^{2}$ Itiro Siio ${ }^{1}$ \\ ${ }^{1}$ Department of Computer Science, Ochanomizu University \\ ${ }^{2}$ Department of Computer Science and Information Engineering, National Taiwan University \\ ${ }^{3}$ Department of Media Architecture, Future University Hakodate \\ \{azusa, siio\}@is.ocha.ac.jp, \{d99922035, hchu\}@csie.ntu.edu.tw, tsuka@acm.org
}

\begin{abstract}
This paper describes the design of a digital fork and a mobile interactive and persuasive game for a young child who is a picky eater and/or easily distracted during mealtime. The system employs Ubicomp technology to educate children on the importance of a balanced diet while motivating proper eating behavior. To sense a child's eating behavior, we have designed and prototyped a sensor-embedded digital fork, called the Sensing Fork. Furthermore, we have developed a story-book and persuasive game, called the Hungry Panda, on a smartphone. This capitalizes on the capabilities of the Sensing Fork to interact with and modify children's eating behavior during mealtime. We report the results of a real-life study that involves mother-child subjects and tested the effectiveness of the Sensing Fork and Hungry Panda game in addressing children's eating problems. Our findings exhibit positive effects for changing children's eating behavior.
\end{abstract}

\section{Author Keywords}

Children, Eating behavior; Food color; Fork; Mobile;

Sensing; Persuasive technology; Ubiquitous computing;

\section{ACM Classification Keywords}

H.5.m. Information Interfaces and Presentation (e.g. HCI): Miscellaneous

\section{INTRODUCTION}

Parents often experience situations where verbal persuasion has only limited effectiveness with their children. A particular case is at mealtime when parents are attempting to instill healthy eating habits in their children. For example, parents often say, "Eat your green food" or "Finish up your plate." Children, however, often have

Permission to make digital or hard copies of all or part of this work for personal or classroom use is granted without fee provided that copies are not made or distributed for profit or commercial advantage and that copies bear this notice and the full citation on the first page. Copyrights for components of this work owned by others than the author(s) must be honored. Abstracting with credit is permitted. To copy otherwise, or republish, to post on servers or to redistribute to lists, requires prior specific permission and/or a fee. Request permissions from Permissions@acm.org.

Ubicomp '14, September 13-17, 2014, Seattle, WA, USA.

Copyright is held by the owner/author(s).Publication rights licensed to ACM. ACM 978-1-4503-2968-2/14/09...\$15.00.

http://dx.doi.org/10.1145/2632048.2632093

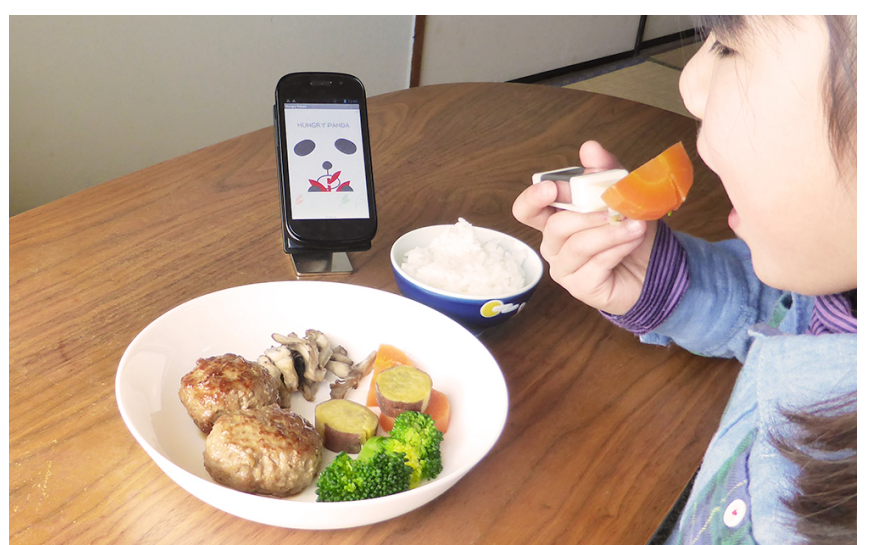

Figure 1. Overview of the Sensing Fork and Hungry Panda game application.

little or no incentive to comply with these directives. Is it possible to apply computing technology to make the eating behavior training experience more enjoyable for children? Several HCI (Human Computer Interaction) projects have explored persuasive technologies [8] for motivating users in diverse daily activities such as brushing teeth, and avoiding a sedentary lifestyle [5], [15]. We believe that the playful aspects of persuasive technology and interaction are especially applicable to children.

A well-balanced diet is an important factor in maintaining good health. From a broader perspective, dietary education today includes how to eat, how to select ingredients, and dietary culture. Because diet and health are closely related, dietary education is and will continue to be a universally important health topic. In many developed countries, governments have been promoting dietary education by providing dietary information on websites $^{12}$ and running dietary classes at schools.

In Japan, owing to concern over increasing unhealthy dietary habits, particularly in unbalanced nutrient consumption, the Dietary Education Basic Law was enacted in 2005. The goal of this legislation was to promote

\footnotetext{
${ }^{1}$ U.S. Department of Agriculture:

http://www.choosemyplate.gov

${ }^{2}$ National Health Service (England):

http://www.nhs.uk/livewell/5ADAY/Pages/5ADAYhome.aspx
} 
healthy dietary habits such that people could enjoy active and healthy lives ${ }^{3}$. Consequently, most kindergarten schools are required to have dietary education classes in which teachers encourage children to eat diverse types of food. However, it is difficult for many parents and children to continue learning and adopting such dietary activities at home because of the lack of time and knowledge. We believe that a new method for dietary education suited to the mealtime period is needed.

We therefore aim to provide an alternative method for motivating children to improve their eating behavior by enhancing a fork, a basic and simple eating utensil. This sensor-embedded fork, called a Sensing Fork, in conjunction with a mobile game, called a Hungry Panda, can facilitate dietary education activities for parents and children at home. To encourage eating a variety of food items, this system makes use of food color, something children can easily identify and understand. By encouraging children to eat foods of different colors, the system teaches children about eating a balanced meal. This simple color-based food selection strategy is an alternative to more complex nutritional lessons that teach different food groups, their nutrition types, functions and roles. Given its simplicity, some nutritionists and physicians recommend a color-based dietary education method [3], that we also adopted in our system. Japanese nutritionists have also proposed the "Five-colored Balanced Food Hygiene," which is expected to be adopted to support children's dietary education [21]. The basic idea is to eat five food colors (RED, WHITE, YELLOW, GREEN, and BLACK) each mealtime. Our color-sensing approach is designed for children following this simple color-based dietary education strategy.

To detect the food color in contact with the fork, we embed a small color sensor at the tip of the Sensing Fork device (Figure 2). The Sensing Fork has other sensory and communication components including three electrodes, a motion sensor, a microcomputer, a Bluetooth radio, and a battery. By processing the sensory data, our system recognizes a child's eating behavior (eating actions and food colors). The sensory data is transmitted to the Hungry Panda game executed on a smartphone. The game is interactive and provides feedback to encourage children to improve their eating behavior.

As a part of the iterative design process, our prior works presented and demonstrated early and preliminary prototypes in [12], [13]. The main contributions of this work are as follows:

1. We implemented a digital fork, called a Sensing Fork embedded with sensors to recognize eating behavior in real time. We explored the feasibility of applying Ubicomp technology to an eating utensil that can enable dietary education and encourage eating behavior for children during daily mealtimes.

\footnotetext{
${ }^{3}$ Cabinet Office, Government of Japan:

http://www8.cao.go.jp/syokuiku/index.html
}

2. We developed a mobile application, Hungry Panda, for young children. The application provides educational material for parents to teach their children about a balanced diet through storytelling at the beginning of the meal. The Hungry Panda game encourages children to eat diverse foods during mealtime.

3. We conducted a nine-day real-life user study with five mother-child pairs to determine whether our system design is feasible for promoting dietary education, healthy eating behavior, and family interaction. We report the findings and, based on the results, we discuss the implications for future designs.

\section{RELATED WORK}

Recently, various projects related to diet have been released in the area of HCI [6]. Grims et al. researched and discussed the possibilities for human-food interaction [9]. Mansour et al. developed a computing game that encourages children and young people to enjoy learning good and bad nutrition balance [17]. Parker et al. proposed two projects: researching the impact on children when nutrition and proper food combinations are displayed in online dietary advertisements [19], and developing a mobile game to explain how to make healthier meal choices for adults [10]. In addition, there are various services that support dietary education, functioning exclusively with the use of a screen ${ }^{4}$.

There have been other projects that address users' eating behavior, such as embedded sensors and computers in the dining place. The Playful Tray targets slow-eating children. As visual feedback, the contents of a dish-type display change based on the amount of food remaining on the dish [16]. Dining Presenter aims to make mealtimes enjoyable and supports dietary education with a projector placed over the table, displaying drawings and text messages made in advance [18]. The Hapifork provides tactile feedback on fast-eating behavior based on the intervals between bites [11]. The EducaTableware [14] developed a fork-type device for children using sound feedback to improve their eating habits. However, these systems did not detect eating behavior effectively because of limitations in the sensor.

Our project therefore developed a fork-type sensing device that embeds various sensors to detect human eating behavior (eating actions and food colors) and a persuasive game application cooperating with the device. Our objective was to make users, especially children, conscious of improving their eating behavior in a natural manner by detecting the user's eating behaviors precisely and providing feedback to the user based on the sensor data obtained.

\footnotetext{
${ }^{4}$ Fatworld: http://www.fatworld.org

${ }^{5}$ Shokuiku Town: http://www.glico.co.jp/shokuiku/game
} 


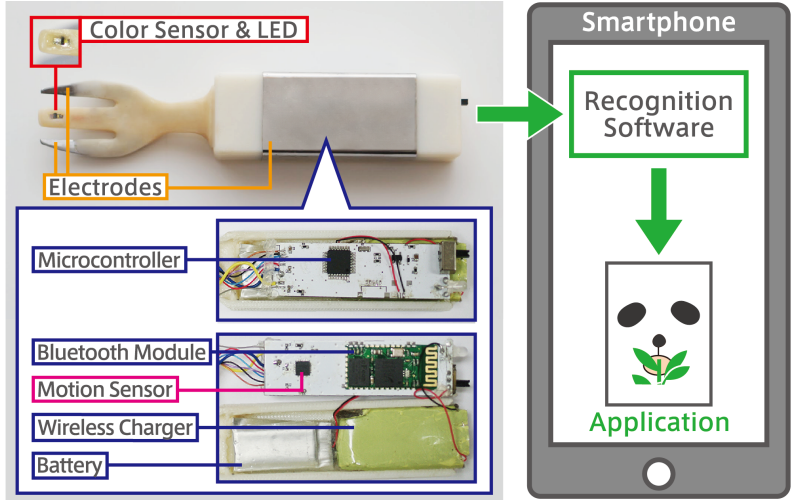

Figure 2. System configuration. Left: The Sensing Fork prototype. Right: The software on the smartphone.

\section{SYSTEM OVERVIEW}

Embedding miniature sensors in utensils enables the detection of natural eating behaviors without introducing new tracking devices and without changing the normal operation and function of the eating utensil. To facilitate interaction with children, the Sensing Fork is designed to work with a mobile device. The Sensing Fork forwards the sensory data to the mobile device. The mobile device then processes the data to recognize the child's eating behaviors associated with the fork. The Hungry Panda game running on the mobile device interacts with the child according to the recognized eating behaviors.

\section{PROTOTYPE DESIGN: SENSING FORK Components of the Sensing Fork}

Most of the electronic components are packaged and hidden inside the fork grip (Figure 2). The controller is an Atmel ATMEGA328P microcontroller. Sensor readings are collected at a rate of ten samples per second and then transmitted to the mobile device through a Bluetooth module. A rechargeable $120 \mathrm{~mA}$ lithium battery powers the Sensing Fork. There is sufficient battery power for the fork to run for two hours. Because it must be washed, the fork is waterproof and includes a wireless charging system. The fork grip is $L 80 \times W 25 \times H 15 \mathrm{~mm}$ in size, making it easily held by small children.

The fork's middle tine contains a tiny breakout board that houses a color sensor and a tiny LED light to illuminate the food contacting the fork. The breakout board is carefully placed between two metal tines and coated with white and transparent resin. The resin is dental grade, ensuring that the fork is washable and safe for eating.

\section{Three-electrode Conductive Probe}

Because both food and the human body are conductive, we leveraged two naive voltage dividers with threeelectrodes to recognize two eating actions: Poking and Biting. As shown in Figure 2 and Figure 3, two metal tines and one metal surface grip are wired to the circuit as electrodes. In the case of Poking, the measured voltage equals the supply voltage when the two fork's tines (electrodes) do not make contact with food. When the two fork tines do contact food, a circuit is created

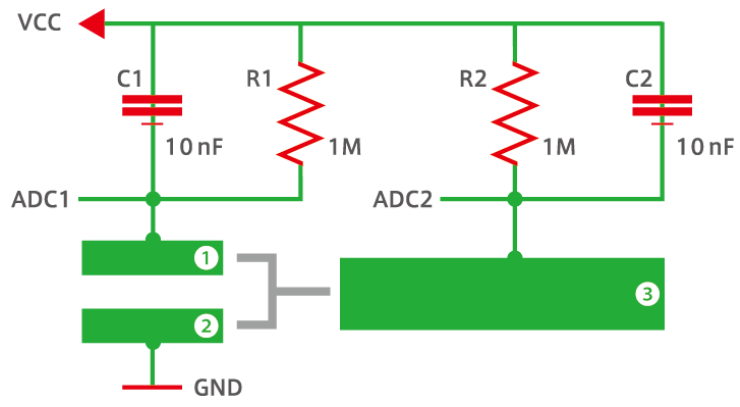

Figure 3. Electrodes and resistance sensing schematic. Marker 1 and 2 represent two fork's tines. Marker 3 is the fork's grip.

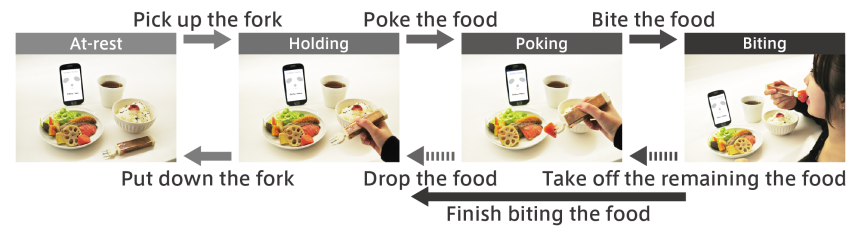

Figure 4. State transition diagram of eating actions.

in which the measured voltage is altered because of the food's impedance. The function for recognizing Biting is similar. When a child's mouth contacts food while holding the fork's grip, i.e., the third electrode, it forms a circuit between the fork's grip and tine, the child's mouth, and the hand. The electrical current that passes through the food and child's body is low and safe.

\section{Six-axis Motion Sensor}

To capture human motion with the fork, the Sensing Fork includes an InvenSense MPU-6050 that integrates a three-axis accelerometer and a three-axis gyroscope into a single chip. The motion data is used to infer changes to the fork's state.

\section{Single-pixel RGB Color Sensor}

The single-pixel RGB color sensor is a TAOS TCS3414 that outputs four-channel color readings (RGB and intensity). It is very small, $L 2.1 \times W 1.9 \times H 0.9 \mathrm{~mm}$. The color sensor samples the food color when a child pokes the food. A tiny white LED next to the color sensor illuminates the food in case the tine penetrates the food too deeply for the sensor to determine the color.

\section{Detecting Eating Actions}

The eating action detector analyzes the sensor readings from the fork and determines the state of the fork during a child's eating action. Figure 4 presents the fork's four states and describes the method used by the proposed system to distinguish between them.

\section{At-Rest}

The fork is At-Rest state when it is not being held by a hand. If the fork is the only eating utensil, this state suggests that the user is not engaging in any eating action. The system is able to infer the At-Rest state given that the accelerometer and gyroscope sensors report no motion. 


\section{Holding}

The fork is in the Holding state when it is being held by the hand without any food on the fork. This state suggests that the user has lifted the fork and may be deciding what food item to grab with the fork. The system infers this Holding state based on the presence of motion from the accelerometer and gyroscope sensors.

\section{Poking}

The fork is in the Poking state when it is being used to poke a food item. The fork's tines contact the food. This state suggests that the user is in the process of selecting food, but has not inserted it into his/her mouth. The system infers the Poking state from two sensor inputs: (1) two-electrode tines measure a non-zero resistance value indicating the presence of food; and (2) the photocell sensor detects food colors. Touching food with the fork triggers the food color recognizer.

\section{Biting}

The fork is in the Biting state when the fork or food on the fork contacts the mouth. This state suggests that the user is biting or tasting the food on the fork. The system infers the Biting state by measuring an altered reading from the fork circuit connecting the child's hand, fork, and mouth.

\section{Classifying Food Types}

Our system uses the food's color as the feature to classify the type of food that contacts the fork. Food type classification includes the following four functions: (1) sample selection, (2) extracting feature, (3) training classifier, and (4) online testing.

\section{Sample Selection}

Because the fork's sampling rate $(10 \mathrm{~Hz})$ is higher than in human's eating actions, our system observes a window of sensor data for each eating action. Consider the case where a child is deciding what to eat next. The child might start by touching one food item before moving on to another, and then another. This can continue until the child sticks the fork tine inside a target food item. Thus, the sensor readings will vary initially and then stabilize when the fork penetrates a food item. To determine this stable window, our system uses a sliding window with a length of five samples and then computes a variance value for this window. If the variance value is lower than the threshold value, our system uses the sensor data in this window for inferring the food type. Note that the fork must also be in the Poking or Biting state.

\section{Extracting Feature}

Four color readings and one resistance value in the sample are used for classifying the food types. The raw color readings can vary between each poking of the same food item. The RGB readings increase linearly with light intensity. Since the ambient lighting may affect the RGB sensory readings, we calculate two-dimensional chromaticity values [2] using the RGB readings as features to mitigate the effect from ambient luminance. Note that chromaticity represents the quality of a color regardless of its luminance. Our system also computes the difference between the RGB values and clear readings as features to train a food detection classifier. When a child is poking a food item, the attached LED on the fork tine becomes the primary light source to illuminate the food's interior color. Based on our experiment, the fork's LED is primary in detecting food color, whereas the ambient light has only a limited effect.

\section{Training Classifier}

A Support Vector Machine (SVM) algorithm is used to train the classifier. The classifier is implemented with LibSVM [4]. The SVM classifier uses the polynomial kernel and one-against-one multiclass classification.

The method used to train the classifier results in differing food classification performance. As the number of food types increases, the performance of the classifier decreases. This is because foods may have similar colors that are ambiguous to the classifier. The classifier can be trained either right before the meal or in pre-training. Training right before the meal (per-meal training) requires the user to collect samples before the meal is about to start. This determines the significant differences between ingredients that are of the same type though not identical. Identical ingredients may still have a slight difference in color. Cooking-style might also change the food's color. A pre-trained classifier is trained prior to any system deployment with food samples that are commonly eaten by children in the community. By comparison, a per-meal classifier is trained with food samples collected from a meal immediately prior to consumption. The advantage to using a pre-trained classifier is that it saves the user from needing to train the classifier. The advantage to using a per-meal classifier is accuracy, because the training samples are identical to the testing samples and because every family serves different food or at least prepares the same food differently, i.e., the same food but with a slightly different color. In other words, the per-meal and pre-trained classifier form a tradeoff between accuracy and convenience in real-life use.

\section{Online Testing}

In our application, the system tests five consecutive samples determined by sample selection. The classifier tests each sample separately. The system reports the results according to the most occurrences of the tested results from the five selected samples. Real-time classification on the Android platform was implemented using the LibSVM library for JAVA. Our application is able to perform online testing smoothly using an Android phone.

Our application classified the food items into five color categories (RED, WHITE, YELLOW, GREEN, and BLACK) according to the "Five-colored Balanced Food Hygiene" [21]. 

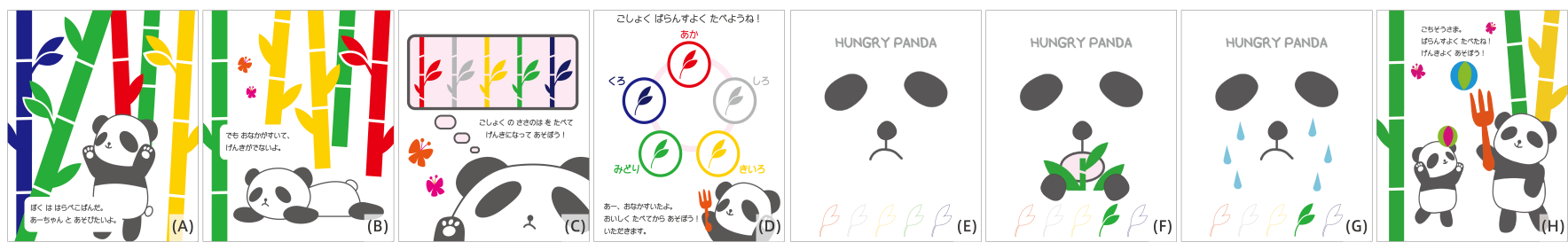

Figure 5. The story and flow of Hungry Panda game application.

\section{PERSUASIVE GAME APPLICATIONS: HUNGRY PANDA}

In this study, we focus on improving a child's dietary behavior, particularly by eating diverse foods with different colors. We developed a persuasive game application, called a Hungry Panda, on a smartphone to be used together with the Sensing Fork. The basic design for the Hungry Panda game has a digital panda imitating the child's eating behavior, including the eating actions and selected food colors. The rationale for designing it this way is to build rapport and foster sympathy for the panda, so that the child wants to help the panda. Because the panda imitates the child's eating behavior, the child is presented with an opportunity and a choice to help the panda by improving his/her own eating behavior. The use of a smartphone is a convenient computing and display device for many people in Japan and elsewhere. We use the panda as a virtual pet, because the panda is familiar to children in most Asian countries. The Hungry Panda game consists of two parts: (1) an interactive picture book for dietary education prior to eating, and (2) an interactive and persuasive game application for collecting points during a meal.

\section{Picture Book for Dietary Education}

It is common for parents to read picture books aloud to their children. Such activities are not only important for educating children but also serve to foster good parentchild relationships. Therefore, we designed a short and simple picture book to teach color-based dietary education. Reading this picture book before a meal helps both parents and children to become familiar with the color-based food game.

The game's first screen is shown in Figure 5 (A), with a panda greeting the child: "Hello, (the child's name). I am Hungry Panda. I want to play with you." This panda greeting is an attempt to initiate friendship with the child and encourage the child's sympathy for the panda. Figure 5 (B) is the second screen that shows a tumbled Hungry Panda: "However, I do not feel perky because I am very hungry." This screen gives a subconscious hint to the child that mealtime is about to begin. Figure 5 (C) presents the third screen: "Let's eat five different colored bamboos to become cheerful." This sets the goal for a properly balanced diet of different food color. Figure 5 (D) shows the next screen "Let's play after the meal. Itadakimasu (Japanese expression of gratitude before meals)." This screen also provides a detailed explanation about the five-colored food items. This short and simple picture book encourages both children and parents to engage in a process that is both fun and entertaining. Because we encourage parents to read the picture book aloud to their children, we removed any vocal narration from the picture book and retain only the background sound effects.

\section{Interactive and Persuasive Game Application}

After the picture book introduction, the unhappy panda appears on the smartphone screen and says, "I am very hungry. I want to eat" (Figure 5 (E)). The panda imitates the child's eating actions, for instance by Holding or Biting, and eats the bamboo grass whose color matches the child's consumed food item (Figure $5(\mathrm{~F})$ ). All consumed food items correspond to the five colors.

This game application specifically targets two common eating problems ${ }^{6}$. Picky Eating: The score (expressed as bamboo grass points and displayed at the bottom of the screen) improves when the user eats food with a different colors. This encourages the child to continue eating to collect more points. At the same time, the child is helping to feed the Hungry Panda. Distraction: The system generates various sounds as an alert to the child. For example, when the child stops eating or puts down the Sensing Fork for a certain time period, the panda cries and exclaims "want to eat more" (Figure $5(\mathrm{G})$ ). When the child picks up the Sensing Fork again, the panda laughs. In the game design, holding the Sensing Fork is not only a way for the child to show an interest in helping, it also encourages the child to concentrate on eating, thereby avoiding distractions. Pressing the finish button produces an animated reward for the child's good behavior - the higher the points obtained, the more impressive the animation (Figure $5(\mathrm{H})$ ).

\section{EXPERIMENTAL EVALUATION}

We conducted two types of experimental evaluation on the Sensing Fork using a smartphone. This section describes the results for detecting eating actions and classifying food colors.

\section{Results for Detecting Eating Actions}

To confirm the ability to detect eating actions, we recruited six participants (three men and women, aged 21-28) and asked them to eat a certain food item, i.e., a bite-size cube of cheese, five times. We instructed each

\footnotetext{
${ }^{6}$ Statistical Surveys conducted by Ministry of Health, Labour and Welfare of Japan in 2005.

http://www.mhlw.go.jp/toukei/list/83-1.html
} 


\begin{tabular}{|c|c|c|c|c|c|c|c|c|c|c|c|c|c|c|c|c|c|c|}
\hline Prediction & $\underset{\Xi}{\Xi}$ & 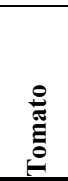 & 音 & 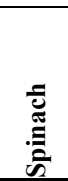 & 芯 & $\stackrel{\square}{\vdots}$ & 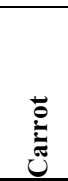 & 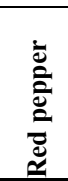 & 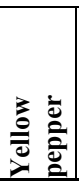 & 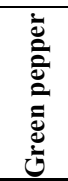 & 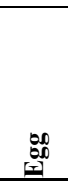 & 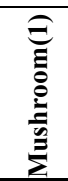 & 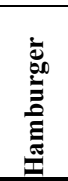 & 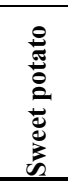 & 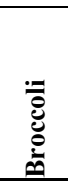 & 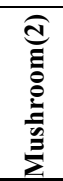 & $\stackrel{\mathscr{E}}{\underline{\underline{\underline{\Sigma}}}}$ & 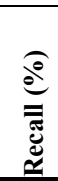 \\
\hline Tuna & 45 & 1 & 0 & 0 & 0 & 0 & 0 & 4 & 0 & 0 & 0 & 0 & 0 & 0 & 0 & 0 & 0 & 90.0 \\
\hline Tomato & 2 & 42 & 0 & 0 & 0 & 0 & 1 & 5 & 0 & 0 & 0 & 0 & 0 & 0 & 0 & 0 & 0 & 84.0 \\
\hline Pumpkin & 0 & 0 & 48 & 0 & 0 & 0 & 0 & 0 & 1 & 0 & 1 & 0 & 0 & 0 & 0 & 0 & 0 & 96.0 \\
\hline Spinach & 0 & 0 & 0 & 43 & 3 & 0 & 0 & 0 & 0 & 3 & 0 & 1 & 0 & 0 & 0 & 0 & 0 & 86.0 \\
\hline Carrot & 0 & 2 & 0 & 0 & 0 & 2 & 45 & 0 & 1 & 0 & 0 & 0 & 0 & 0 & 0 & 0 & 0 & 90.0 \\
\hline Red pepper & 1 & 6 & 0 & 0 & 1 & 0 & 0 & 42 & 0 & 0 & 0 & 0 & 0 & 0 & 0 & 0 & 0 & 84.0 \\
\hline Yellow pepper & 0 & 0 & 3 & 0 & 1 & 0 & 0 & 0 & 43 & 0 & 0 & 0 & 3 & 0 & 0 & 0 & 0 & 86.0 \\
\hline Green pepper & 0 & 0 & 0 & 4 & 3 & 0 & 0 & 0 & 0 & 41 & 0 & 0 & 0 & 0 & 1 & 1 & 0 & 82.0 \\
\hline Egg & 0 & 0 & 2 & 0 & 0 & 0 & 0 & 0 & 0 & 0 & 48 & 0 & 0 & 0 & 0 & 0 & 0 & 96.0 \\
\hline Mushroom(1) & 0 & 0 & 0 & 3 & 0 & 0 & 0 & 0 & 0 & 0 & 0 & 46 & 0 & 0 & 1 & 0 & 0 & 92.0 \\
\hline Rice & 0 & 0 & 0 & 0 & 0 & 0 & 0 & 0 & 0 & 0 & 0 & 0 & 0 & 0 & 0 & 0 & 50 & 100 \\
\hline Precision (\%) & 93.8 & 82.4 & 90.6 & 75.4 & 77.0 & 73.6 & 97.8 & 82.4 & 87.8 & 82.0 & 98.0 & 95.8 & 73.5 & 89.3 & 95.2 & 98.0 & 100 & \\
\hline
\end{tabular}

Table 2. The confusion matrix of classifying food types. (1)Shiitake Mushroom. (2) Eryngii Mushroom.

\begin{tabular}{|c|c|c|c|c|c|c|c|}
\hline \multirow{2}{*}{ Action } & \multicolumn{6}{|c|}{ Participant } & \multirow{2}{*}{ Avg. } \\
\hline & $\overline{\mathbf{A}}$ & $\bar{B}$ & $\begin{array}{l}\mathbf{C} \\
\end{array}$ & D & $\mathbf{E}$ & $\mathbf{F}$ & \\
\hline Holding-1 & $\begin{array}{c}100 \% \\
(0.3)\end{array}$ & $\begin{array}{c}100 \% \\
(0.5)\end{array}$ & $\begin{array}{c}100 \% \\
(0.3)\end{array}$ & $\begin{array}{c}100 \% \\
(0.5)\end{array}$ & $\begin{array}{c}100 \% \\
(0.6)\end{array}$ & $\begin{array}{c}100 \% \\
(0.4)\end{array}$ & $\begin{array}{c}100 \% \\
(0.4)\end{array}$ \\
\hline Poking & $\begin{array}{c}100 \% \\
(0.6)\end{array}$ & $\begin{array}{c}100 \% \\
(0.3)\end{array}$ & $\begin{array}{c}100 \% \\
(0.9)\end{array}$ & $\begin{array}{c}100 \% \\
(0.6)\end{array}$ & $\begin{array}{c}100 \% \\
(0.6)\end{array}$ & $\begin{array}{c}100 \% \\
(0.5)\end{array}$ & $\begin{array}{c}100 \% \\
(0.6)\end{array}$ \\
\hline Biting & $\begin{array}{c}100 \% \\
(0.3)\end{array}$ & $\begin{array}{l}80 \% \\
(0.9)\end{array}$ & $\begin{array}{l}60 \% \\
(0.9)\end{array}$ & $\begin{array}{l}80 \% \\
(1.2)\end{array}$ & $\begin{array}{l}80 \% \\
(1.1)\end{array}$ & $\begin{array}{l}60 \% \\
(0.7)\end{array}$ & $\begin{array}{l}77 \% \\
(0.9)\end{array}$ \\
\hline Holding-2 & $\begin{array}{c}100 \% \\
(1.1)\end{array}$ & $\begin{array}{c}100 \% \\
(1.8)\end{array}$ & $\begin{array}{c}100 \% \\
(1.5)\end{array}$ & $\begin{array}{c}100 \% \\
(1.1)\end{array}$ & $\begin{array}{c}100 \% \\
(0.5)\end{array}$ & $\begin{array}{c}100 \% \\
(1.2)\end{array}$ & $\begin{array}{c}100 \% \\
(1.2)\end{array}$ \\
\hline At-Rest & $\begin{array}{c}100 \% \\
(1.5)\end{array}$ & $\begin{array}{c}100 \% \\
(1.4)\end{array}$ & $\begin{array}{c}100 \% \\
(1.5)\end{array}$ & $\begin{array}{c}100 \% \\
(1.5)\end{array}$ & $\begin{array}{c}100 \% \\
(1.1)\end{array}$ & $\begin{array}{c}100 \% \\
(1.4)\end{array}$ & $\begin{array}{c}100 \% \\
(1.4)\end{array}$ \\
\hline
\end{tabular}

Table 1. The accuracy of detecting each eating action. The numbers in parentheses denote the average time (sec.) required to detect each eating action.

participant to put the Sensing Fork back on the table to examine the recognition accuracy for the completion of a single sequence in eating actions. Each eating sequence included Holding-1, Poking, Biting, Holding-2, and At-Rest, in that order. Holding-2 was the time when the fork left the participant's mouth. Each participant repeated the above eating sequence five times. All participants were using the Sensing Fork for the first time. We did not describe the system to them in advance.

Table 1 shows the accuracy of eating action detection and the average time required for detection. The overall recognition rate was 95\%. However, the Biting recognition rate was only $77 \%$. Biting was falsely detected as Holding-2. On observing the falsely recognized Biting action on the video, we noticed that the average time taken was only 0.1s, whereas a correctly recognized Biting lasted around $0.9 \mathrm{~s}$. Thus, when the fork touched the participant's mouth for only a brief moment, it was incorrectly recognized. This problem stems from the lim-

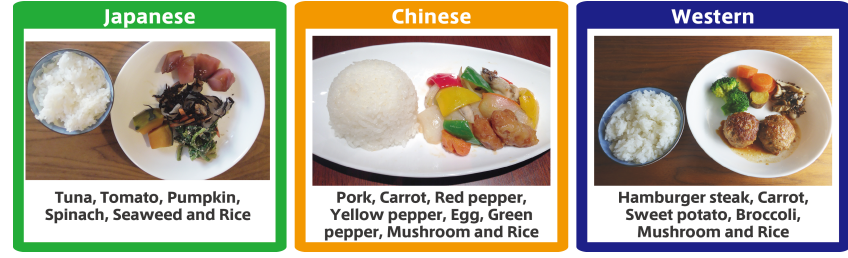

Figure 6. The examples of food items in three cuisines.

itation in the implementation that set the recognition interval as 0.1s. This interval was set low in order to conserve battery power. We believe that the recognition rate will be much improved by modifying the interval.

As shown in Table 1, the average time for detecting the eating state was 0.9s. Notably, however, detecting AtRest required $1.4 \mathrm{~s}$. This is because the fork infers it is At-Rest when it remains motionless for more than one second. However, in general, actions such as putting down and lifting the fork cannot be repeated at high speeds and therefore this is not considered a problem. Further, Holding-2 required an average of $1.2 \mathrm{~s}$. This resulted from a problem with the experimental program. Specifically, a sample animation of around 2.0s was displayed after the Biting action and the state did not update until the end of the animation. The actual detection time is thought to be equivalent to Holding-1.

\section{Results of Classifying Food Types}

To examine the accuracy in classifying the food types, we collected food items from three different cuisines: Japanese, Chinese, and Western cuisines typical in Japanese households (Figure 6). Each cuisine included multiple food types providing a balanced diet. 
We collected food samples using the Sensing Fork to perform a poking action with the food item. Based on the three different cuisines, we collected 17 types of food items and 50 samples of each, because the color of the same ingredient could change with the cooking method. In each experiment, we poked the food item ten times. The collected dataset contained 850 samples from the 17 food types. We conducted a 10 -fold cross-validation to evaluate the F-measure accuracy of the food-color classifier. Each round of cross-validation randomly divided all the samples into a training set and testing set. The overall F-measure accuracy was $87.5 \%$ in this experiment. Table 2 shows the confusion matrix for the result that evaluated classifying 17 food types under the cross-validation. Improper poking caused a few incorrect classifications. Most incorrect classifications were caused by foods with similar colors from the sensor's viewpoint. For example, pork and hamburger steak, yellow pepper and pumpkin, tuna and red pepper, and carrot and tomato were similar groupings. We further classified the 17 food types into three categories - Japanese, Chinese, and Western - and evaluated their F-measure accuracy individually. Because individual cuisine has less ambiguous food types, the accuracies of the Japanese, Chinese, and Western cuisines were $94.7 \%, 96.2 \%$, and $93.8 \%$, respectively. Samples from the three cuisines were also used in our user study with the children. The food samples were not only used to evaluate the performance of the Sensing Fork but also to train the classifiers used in the Hungry Panda game during the user study.

\section{USER STUDY}

We conducted a real-life user study involving five motherchild pairs who tested the Sensing Fork and Hungry Panda game at home. This study assessed how effective our system performed in residential settings. This study also evaluated how effective our system addressed children's picky eating and distraction problems and the effectiveness of the system's dietary education for the mother-child pairs.

\section{Procedure}

Table 3 shows the profiles of the five mother-child pairs, including the age/gender of each child, the child's eating problem, and the child's disliked foods as reported by the child's mother. All participants were Japanese, living in Japan. We denote each child as Child-A, ..., Child-E and each mother as Mother-A, ..., Mother-E. Child-A and Child-D are siblings. That is, the mother of Child-D is also Mother-A. All the children used our system for the first time and did not receive official instructions for the system. We did explain the procedure for the user study and provided a user study set to the mothers directly. The user study set contained a Sensing Fork with a wireless charger, a smartphone with a charger, a diary, and the basic instruction booklet.

The user study consisted of two parts: a nine-day real-life user study and a post-meal survey. In the user study, we

\begin{tabular}{|c|c|c|c|c|}
\hline Participant & Age & Gender & Eating problem & Dislike food \\
\hline Child-A & 2 & Boy & Picky eating & $\begin{array}{c}\text { General } \\
\text { vegetable }\end{array}$ \\
\hline Child-B & 3 & Girl & $\begin{array}{c}\text { Picky eating, } \\
\text { Distraction }\end{array}$ & $\begin{array}{c}\text { General } \\
\text { vegetable }\end{array}$ \\
\hline Child-C & 5 & Girl & $\begin{array}{c}\text { Picky eating, } \\
\text { Distraction }\end{array}$ & Mushroom \\
\hline Child-D & 7 & Girl & Picky eating & Green pepper \\
\hline Child-E & 8 & Girl & Picky eating & $\begin{array}{c}\text { General } \\
\text { vegetable }\end{array}$ \\
\hline
\end{tabular}

Table 3. Details of child participants.

asked each mother to take pictures of the dinner plates before and after meals, to take videos during mealtime, and to note comments and impressions in a diary after eating. To determine what effect our system has on eating habits, the children used our system on days 4-6 (Term 2) and used a regular fork on days 1-3 (Term 1) and 7-9 (Term 3 ). We also asked the mothers to make three kinds of meals according to the cuisines used in the experimental evaluation: Japanese, Chinese, and Western. We specified the main dish (sashimi tuna, sweet-and-sour pork, and hamburger steak) and asked them to include five colored food items (RED, WHITE, YELLOW, GREEN, and BLACK) as well as foods that the child dislikes. To compare the eating behavior of each participant during the different terms fairly, participants repeated each cuisine three times. For example, participants would eat Japanese on the 1st, 4th, and 7th day, Chinese on the 2nd, 5th, and 8th day, and Western on the 3rd, 6th, and 9 th day. The cooking-style and ingredient selection were not strictly managed. For instance, some mothers put tomato sauce and others put demi-glace sauce on their hamburger steaks. To avoid the disturbing the dining atmosphere, we were physically absent during the user study. A camera was setup to record videos of all meal sessions. In the post-meal survey, we asked the mothers to complete a questionnaire and participate in a onehour oral interview. The questions and results of the questionnaire are summarized in Table 4.

\section{Results and Findings}

This section presents results and findings by analyzing data collected from the user study. The collected data include recorded mealtime videos and semi-structured interviews answered by the mothers on their experiences of the nine-day user study. After transcribing the recorded mealtime videos and the interviews, we coded transcriptions and organized the themes of the findings based on thematic analysis [1].

Results from the post-meal Question I suggested that our system made a positive impression on all motherchild pairs. Results from Question II indicated that most children had no problem holding and poking food with the Sensing Fork. One noticeable exception is ChildA, who was only two years old at the time and found that the fork grip was larger than he was accustomed to. Despite this difficulty, Child-A was able to finish his meals using the Sensing Fork. 


\begin{tabular}{|c|c|c|c|c|c|c|}
\hline \multicolumn{2}{|r|}{ Question } & $\mathbf{A}$ & B & C & D & $\mathbf{E}$ \\
\hline & $\begin{array}{l}\text { How do you and your child feel about overall impression of our system? } \\
\text { (1: hate - 3: neutral - 5: like) }\end{array}$ & 5 & 5 & 5 & 5 & 4 \\
\hline & $\begin{array}{l}\text { Is the Sensing Fork easy to hold and poke for your child? } \\
\text { (1: difficult - 3: neutral - 5: easy) }\end{array}$ & 2 & 5 & 5 & 4 & 3 \\
\hline & $\begin{array}{l}\text { How did you feel about recognition accuracies of eating actions and colors? } \\
\text { (1: poor - 3: neutral - 5: good) }\end{array}$ & 3 & 4 & 3 & 3 & 3 \\
\hline & $\begin{array}{l}\text { Did our system affect your child's eating behavior? } \\
\text { (1: negative - 3: neutral - 5: positive) }\end{array}$ & 4 & 4 & 5 & 5 & 4 \\
\hline & $\begin{array}{l}\text { Did you and your child feel uncomfortable when our system was on the dining table? } \\
\text { (1: much - 3: neutral - 5: not at all) }\end{array}$ & 3 & 5 & 5 & 3 & 5 \\
\hline & $\begin{array}{l}\text { Do you think our system might disturb the communication among family members? } \\
\text { (1: much - 3: neutral - 5: not at all) }\end{array}$ & 5 & 5 & 5 & 5 & 5 \\
\hline VII. & $\begin{array}{l}\text { How did you and your child feel when the panda cried? } \\
\text { (1: negative - 3: neutral - 5: positive) }\end{array}$ & 5 & 4 & 5 & 5 & 4 \\
\hline
\end{tabular}

Table 4. The results of the questionnaire in the post-meal survey (five-point Likert scale). Higher score is better.

\section{System Accuracies in the Real-World}

The post-meal Question III attempts to understand how well the participating mothers perceive the fork's sensing accuracy and whether it is good enough for playing the Hungry Panda game. Later, we conducted semistructured interviews to further investigate the mothers on their experiences with the system's recognition accuracy. All mothers said that the eating action detection was near perfect; however, they observed that the food color classification was sometimes incorrect and inaccurate food classification was the primary reason behind their 3-4 scores in Question III.

To quantify the accuracy of food color classification, we analyzed the recorded mealtime videos from the meal sessions. A research-trained staff coded the recorded mealtime videos and labeled the ground-truth food poked by children. We then calculated the classification accuracy by comparing the ground-truth data with the output from the food-color classifier. The average food classification accuracy was $52.3 \%$, significantly lower than the $87.5 \%$ accuracy in the pre-study performance evaluation. There were several reasons for this accuracy gap. The mothers used a pre-trained classifier that is less accurate than a per-meal classifier. Some children added colored seasonings to the meals, e.g., soy sauce on sashimi tuna, resulting in significant color changes to the food. Although mothers noticed the classification errors had an adverse effect on the system, none of them reported frustration or indicated that these classification errors rendered our system unusable. For example, Mother-A said: "The accuracy of classifying the food color was not indispensable because Child-A only knows the names of a few colors. He seemed to enjoy the panda acting the way that he did." Mother-B and Mother-C gave similar remarks.

\section{Improvement in Picky Eating}

Results from Question IV suggested most children improved their eating problems than usual. Table 3 shows that all participating children were picky eaters. During

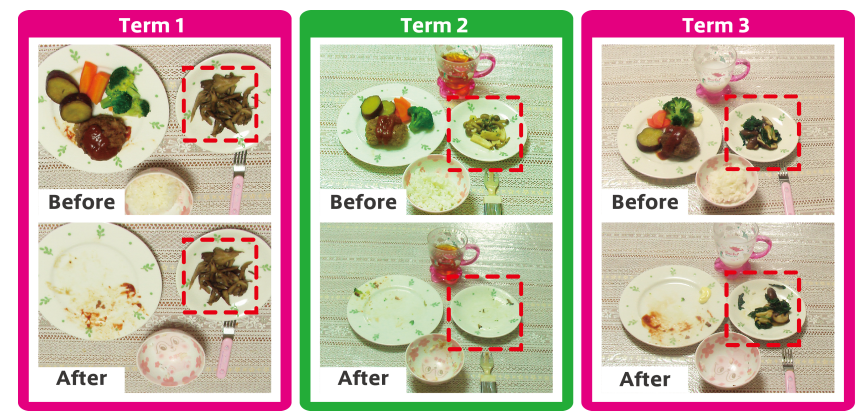

Figure 7. Child-C's dishes before and after of Western food days for each term.

the interviews, we asked the mothers how the Hungry Panda game affected their children's picky eating. Most of them responded positively. For example, Mother-A responded, "If we (my husband or I) encourage Child-A to eat vegetables, he almost never does. However, he ate some vegetables without our persuasion during the Hungry Panda game. Also, Child-D ate all her green pepper, something she used to hate." Child-B was strongly disinclined to eat red pepper on the Chinese food day in Term 1 even her mother encouraged her. However, Child-B ate red pepper in Term 2, despite disliking them. Mother-C shared a similar experience: "On the Western food day in Term 1, my child would not eat the mushrooms at all; however, in Term 2, she ate all her mushrooms without much persuasion from me. I was very surprised. In Term 3, she again ate most of the mushrooms." Figure 7 shows pictures of Child-C's Western food day plate before and after meals.

These examples suggest that our system was able to help children overcome their aversion to certain foods. In the cases of Child-A, Child-B and Child-C, their mothers noted that the reason for their children's dislike was that they had never tried the food or they were turned off by the food's appearance. However, we could not see remarkable effects on Child-E. From the recorded mealtime videos, we observed that Child-E and Mother-E 
interacted much less than the other participating families. During mealtime, they were mainly talking about daily life rather than immersing themselves in the game.

\section{Improvement in Distraction}

Child-B and Child-C have a distraction problem, meaning that they cannot concentrate on eating and often leave the table to play with toys nearby (Table 3 ). Results from Question IV of Child-B and Child-C includes their distraction problem results besides picky eating. Both mothers observed that they were able to generate their children's sympathy toward the Hungry Panda, as a means to persuade their children to concentrate on eating. During the interview, Mother-B said: "Child-B really does not like to eat, and so it always takes a long time to begin. However, during Term 2, she sat earlier than usual by the panda on the table. Moreover, the Hungry Panda picture book included her name, so this improved her interest and concentration. I could make her pay attention to the meal by saying: "Look! The panda is crying because he is very hungry. If you eat, the panda will be happy!" Normally, she does not listen to what I say; however I found that if I said things through the panda, she would listen." The recorded mealtime videos revealed the following interaction between Mother-C and Child-C on the Chinese food day in Term 2. Child-C placed the Sensing Fork on the table for a while, and then the panda said, "I want to eat more...(crying)" MotherC said, "Miss Panda wants you to eat more! Haha!" Child-C then picked up the Sensing Fork and continued eating.

These findings suggest that the Hungry Panda game was successful in attracting children's attention. This is vital. If a game cannot attract attention and interest in the first place, it is not possible to take it to the next step in persuading children to improve their eating behavior.

\section{Potential Effect of Dietary Education}

During the interview, we asked the mothers about how effective was the system's dietary education on family member. All mothers agreed that reading the picture book prior to eating a meal was a natural prelude to the meals. Mother-A said: "I originally thought that dietary education might be a little too difficult for Child-A. Of course, it was the first time educating him about diet, but he could pick up and understand little pieces. In addition, after nine days of reading the picture book, Child-D remembered and recited the five food colors." Mother-A further commented: "From using this system, I felt that my cooking did not have as many diverse food items as it should. I will prepare more diverse food items in the future." Mother-B said: "Child-B cannot read and write Japanese, but she could remember the five colors during eating and tried to collect five food colors during Term 2. I could also learn the proper balanced diet by using this system. Because she has not started school yet, we did not know about dietary education." Mother-E commented: "Child-E learns dietary education at school, and she finishes all her school lunches. But at home, because
I do not force her with anything, she acts spoiled and does not eat food she dislikes. The Hungry Panda game was good that she also eats such disliked foods at home as well."

Our findings suggest that the Hungry Panda's picture book was easy to learn as a dietary education tool, and it affected the behavior and mind of mother-child pairs.

\section{Effect of Mobile Device at the Dining Table}

The post-meal Questions V and VI attempt to address any potentially negative effects in having a smartphone at the dining table during meals. Three mothers did not express any discomfort because they always put their smartphones in a visible place in the dining place. During the interview, Mother-A raised a concern about the potential conflict between the TV and a smartphone during mealtime. Mother-A said: "The reason for giving the score 3 on Question $V$ is this: If mealtime includes all family members, I turn off the TV. In this case, the system may interrupt our conversation. When my children eat by themselves or alone, I turn on the TV. If the smartphone is an extension of the TV, I can accept it. Also, the Sensing Fork and Hungry Panda game provide some meaningful education, so now I like it." Mother-C noted that smartphones were acceptable when used only as a display during mealtime: "If my child touches and fiddles with the smartphone during mealtime, I will be angry. However, in Term 2, the smartphone was merely a display; therefore, I did not mind it."

\section{Effect of Crying Panda as Negative Feedback}

The post-meal Question VII aims to understand how participants perceive the use of the crying panda and whether this negative reinforcement is counter-productive to improving their children's eating behavior. Recall that the crying panda appears when a child stops eating or puts down the Sensing Fork for a certain time period. Questionnaire results report nothing negative about the crying panda. During the interview, we investigated this question further with the mothers. They responded that they did not mind the crying panda.

\section{Limitations in Feeding}

It is common for parents to feed very small children their food directly. However, our system is not intended for assisted feeding owing to the design of the mechanism. Although the panda recognizes Holding and Poking, the system will not recognize Biting when the parent is holding the Sensing Fork; hence, the child cannot gain any bamboo grass points.

From the recorded mealtime videos, we found that our system has the possibility to encourage small children to feed themselves owing to this limitation. Mother-A (to Child-A), Mother-B, and Mother-C sometimes feed food items to their child directly. Child-C sometimes asked Mother-C to feed her. However, they soon understood the system's limitation because the panda did not eat the bamboo grass (and only held it). Mother-C then stimulated Child-C to eat by herself, saying, "If you eat 
by yourself, the panda will be more happy." Following this, Child-C tried to eat by herself.

\section{Dual Sensing Forks Scenario}

During the interview, Mother-A stated, "Child-D wanted to reach the same bamboo grass points that Child-A had already obtained. Then, Child-D attempted to compete with Child-A." Although we did not design our system considering a multiple-user scenario, we observed this interesting interaction between Child-A and Child-D.

\section{DISCUSSION}

\section{Importance of Parental Involvement}

Our device is not intended to replace parents and their role in rearing children. Instead, our system is designed to be an assistive device to help parents generate positive mealtime interaction with their children. The game uses the Hungry Panda, a charming virtual pet, to stimulate parent-child conversations and to provide opportunities for parents to draw out children's motivation to eat. For example, when Child-C put down the Sensing Fork causing the panda to cry, Mother-C used this opportunity to motivate her child to eat: "Miss Panda wants to eat more! Haha!" and Child-C responded positively by picking up the Sensing Fork to help the Hungry Panda. In the user study, we have observed most mothers treating the game as an opportunity for motivating their children to eat. As an assistive device, our system should not offer excessive feedback that may draw too much attention to the game, interfering with parent-child interaction. We observed that if a child interacted with our system without parental involvement, our device was not effective for improving eating habits.

\section{Use of Negative Feedback}

Although prior works [7][15] cautioned against the use of negative feedback, suggesting that it may adversely lose the user's drive for behavioral change, results from our user study indicate that mothers did not mind the negative aspects in the crying panda. As a matter of fact, mothers were able to use the opportunities presented by the crying panda to stimulate their children to eat. We believe that there is some space for the use of negative feedback with children, and that negative feedback ought to be designed carefully to mitigate any undesirable effects. In our case, the negative effect of the crying panda is only temporary - rapidly switching to positive feedback, a happy panda, when the child again picks up the Sensing Fork. This rapid switch from negative to positive feedback encourages children to want to improve their eating habits in order to fulfill their desire help the Hungry Panda.

\section{Multiple-user Scenario}

We believe that a multiple-user system can capitalize on children's competitive attitude to further enhance motivation. However, multiple-user competition may not work for very young children as most children develop competitive attitudes at around the age of four [20]. Our user study involved a two-children scenario. We observed competitive tendencies in the eight years old, Child-D, but not in the two years old, Child-A.

\section{LIMITATIONS}

Food Classification: Pre-trained or Per-meal Classifier

A pre-trained classifier provides low food-classification accuracy but requires no user effort to train the classifier. A per-meal classifier provides high food-classification accuracy but requires some user effort to train the classifier. In consideration for the parent, we adopted a pre-trained classifier in the real-life user study - hence the negative feedback from the mother respecting classification error. To reduce the effort involved in training a per-meal classifier, we have created a step-by-step tool in the application with which a parent can poke each food sample in his/her child's meal multiple times using the Sensing Fork. Upon completion, the application will automatically send the food-sample data for training and build a meal-specific classifier.

\section{Fork, Spoon and Other Utensils}

To eat food such as soups or stews, a spoon is a more appropriate utensil than a fork. It is possible to reuse the design for the Sensing Fork and apply it for building other Sensing Utensils. For example, motion sensors embedded in a Sensing Spoon can recognize spoon actions. A pair of electrodes can be used to distinguish liquid from solid food, because liquids have a lower resistance value than solids.

\section{Game Application Design for Persuasion and Education}

With repeated use, children may become weary of an unchanging picture book and game. However, because the picture book and game are mobile applications, it is relatively easy for users to download new educational content in the picture book and/or update the Hungry Panda game with a new game that a child has not yet played.

\section{CONCLUSION}

We implemented a sensor-embedded eating utensil called the Sensing Fork for detecting a user's eating behavior (eating actions and food colors). As a sample application compatible with the Sensing Fork, we designed the Hungry Panda game targeting children's eating behavior. The aim of the Hungry Panda game was to address children's eating issues, including picky and distracted eating. We conducted a real-life user study to demonstrate that our system can enable persuasive technology at the dining table to improve children's eating behavior. Embedding the sensing technology into a conventionally-used item is more acceptable to the children. We conclude that children's eating habits can be improved if persuasive education tools are included in daily life.

\section{ACKNOWLEDGMENTS}

This work was supported by Grant-in-Aid for JSPS Fellows Grant Number 25-10205 and JST PRESTO Program. The authors appreciate the support of participating labmembers and families. 


\section{REFERENCES}

1. Braun, V., and Clarke, V. Using thematic analysis in psychology. Qualitative Research in Psychology 3, 2 (2006), 77-101.

2. Broadbent, A. D. A critical review of the development of the cie1931 rgb color-matching functions. Color Research and Application 29, 4.

3. Broadbent, Arthur D. Blackburn, G. L., and Waltman, B. A. Physician's guide to the new 2005 dietary guidelines: how best to counsel patients. Cleveland Clinic Journal of Medicine 72, 7 (2005), 609-618.

4. Chang, C.-C., and Lin, C.-J. Libsvm: A library for support vector machines. ACM Trans. Intell. Syst. Technol. 2, 3, (2011), 27:1-27:27.

5. Chang, Y.-C., Lo, J.-L., Huang, C.-J., Hsu, N.-Y., Chu, H.-H., Wang, H.-Y., Chi, P.-Y., and Hsieh, Y.-L. Playful toothbrush: ubicomp technology for teaching tooth brushing to kindergarten children. In Proceedings of the SIGCHI Conference on Human Factors in Computing Systems, CHI '08, ACM (2008), 363-372.

6. Comber, R., Ganglbauer, E., Choi, J. H.-J., Hoonhout, J., Rogers, Y., O'Hara, K., and Maitland, J. Food and interaction design: designing for food in everyday life. In $\mathrm{CHI}$ '12 Extended Abstracts on Human Factors in Computing Systems, CHI EA '12, ACM (2012), 2767-2770.

7. Consolvo, S., McDonald, D. W., Toscos, T., Chen, M. Y., Froehlich, J., Harrison, B., Klasnja, P., LaMarca, A., LeGrand, L., Libby, R., Smith, I., and Landay, J. A. Activity sensing in the wild: A field trial of ubifit garden. In Proceedings of the SIGCHI Conference on Human Factors in Computing Systems, CHI '08, ACM (2008), 1797-1806.

8. Fogg, B. J. Persuasive technology: using computers to change what we think and do. Ubiquity 2002, December (2002).

9. Grimes, A., and Harper, R. Celebratory technology: new directions for food research in hci. In Proceedings of the SIGCHI Conference on Human Factors in Computing Systems, CHI '08, ACM (2008), 467-476.

10. Grimes, A., Kantroo, V., and Grinter, R. E. Let's play!: Mobile health games for adults. In Proceedings of the 12th ACM International Conference on Ubiquitous Computing, Ubicomp '10, ACM (2010), 241-250.

11. HAPILABS. Hapifork. http://www.hapilabs.com.

12. Kadomura, A., Li, C.-Y., Chen, Y.-C., Chu, H.-H., Tsukada, K., and Siio, I. Sensing fork and persuasive game for improving eating behavior. In Proceedings of the 2013 ACM Conference on Pervasive and
Ubiquitous Computing Adjunct Publication, UbiComp '13 Adjunct, ACM (2013), 71-74.

13. Kadomura, A., Li, C.-Y., Chen, Y.-C., Tsukada, K., Siio, I., and Chu, H.-H. Sensing fork: Eating behavior detection utensil and mobile persuasive game. In CHI '13 Extended Abstracts on Human Factors in Computing Systems, CHI EA '13, ACM (2013), 1551-1556.

14. Kadomura, A., Tsukada, K., and Siio, I. Educatableware: Computer-augmented tableware to enhance the eating experiences. In CHI '13 Extended Abstracts on Human Factors in Computing Systems, CHI EA '13, ACM (2013), 3071-3074.

15. Lin, J. J., Mamykina, L., Lindtner, S., Delajoux, G., and Strub, H. B. Fish'n'steps: Encouraging physical activity with an interactive computer game. In Proceedings of the 8th International Conference on Ubiquitous Computing, UbiComp'06, Springer-Verlag (2006), 261-278.

16. Lo, J.-L., Lin, T.-Y., Chu, H.-H., Chou, H.-C., Chen, J.-H., Hsu, J. Y.-J., and Huang, P. Playful tray: adopting ubicomp and persuasive techniques into play-based occupational therapy for reducing poor eating behavior in young children. In Proceedings of the 9th international Conference on Ubiquitous Computing, UbiComp '07, Springer-Verlag (2007), $38-55$.

17. Mansour, A., Barve, M., Bhat, S., and Do, E. Y.-L. Munchcrunch: a game to learn healthy-eating heuristics. In Proceedings of the 8th International Conference on Interaction Design and Children, IDC '09, ACM (2009), 166-169.

18. Mori, M., Kurihara, K., Tsukada, K., and Siio, I. Dining presenter: Augmented reality system for a dining tabletop. In Proceedings of the 2009 ACM Conference on Ubiquitous Computing Supplemental Publication, UbiComp '09 Supplemental, ACM (2009), 168-169.

19. Parker, A. G., McClendon, I., Grevet, C., Ayo, V., Chung, W., Johnson, V., and Mynatt, E. D. I am what i eat: identity \& critical thinking in an online health forum for kids. In Proceedings of the SIGCHI Conference on Human Factors in Computing Systems, CHI '13, ACM (2013), 2437-2446.

20. Priewasser, B., Roessler, J., and Perner, J. Competition as rational action: Why young children cannot appreciate competitive games. Journal of Experimental Child Psychology 116, 2 (2013), $545-559$.

21. Sugimoto, K. Fun and Delicious Five-colored Balanced Food Hygiene: For Preventing and Overcoming of Lifestyle Diseases (in Japanese). Footwork Publishers, 1998. 